\title{
La neurodidáctica como una herramienta pedagógica en la praxis de los docentes integrales de Educación General Básica Elemental
}

\author{
Fecha de recepción: 2021-09-20 • Fecha de aceptación:2021-11-29 • Fecha de publicación: 2022-01-10
}

Carolina Estefanía Saquicela Richards Unidad Educativa Particular "Julio María Matovelle", Ecuador caroes2097@hotmail.com https://orcid.org/0000-0002-1145-4011

\section{Resumen}

Hoy en día, los docentes deben modificar sus estrategias metodológicas basándose en el funcionamiento básico del cerebro del educando. Por ello, la pregunta principal de esta investigación se genera en ¿qué significados emergen de la experiencia vivida de los docentes de Educación General Básica (EGB) Elemental, en cuanto a la aplicación de la neurodidáctica como mecanismo de mejoramiento de la praxis de enseñanza- aprendizaje? Para lo cual, el objetivo principal fue comprender los significados que emergen de la experiencia vivida de los docentes de EGB Elemental, en relación con lo comentado como parte de la pregunta inicial. A su vez, se desprenden cuatro objetivos que se relacionan con las inteligencias múltiples, las estrategias metodológicas y neuroeducativas que el equipo docente utiliza en su praxis educativa. La metodología se basó en el enfoque cualitativo, obteniendo puntos favorables en la aplicación de la neurodidáctica en educación, para todas las edades. Como parte de las conclusiones se determina que esta nueva herramienta pedagógica está a la vanguardia de la educación, porque está pensada en cada habilidad del educando. Tal es así, que da la oportunidad de trabajar los mismos temas de la malla curricular que el Ministerio de Educación establece. 
Palabras clave: cerebro, estrategias neuroeducativas, neurociencias, neurodidáctica, praxis docente

\begin{abstract}
Nowadays, teachers must modify their methodological strategies based on the basic functioning of the learner's brain. Therefore, the main question of this research is generated in what meanings emerge from the lived experience of Elementary General Basic Education (EGB) teachers, regarding the application of neurodidactics as a mechanism for improving the teaching-learning praxis? For which, the main objective was to understand the meanings that emerge from the lived experience of Elementary General Basic Education (EGB) teachers, in relation to what was commented as part of the initial question. In turn, four objectives related to multiple intelligences, methodological and neuroeducational strategies used by the teaching team in their educational praxis were derived. The methodology was based on the qualitative approach, obtaining favorable points in the application of neurodidactics in education, for all ages. As part of the conclusions, it was determined that this new pedagogical tool is at the forefront of education, because it is designed for each learner's ability. Thus, it gives the opportunity to work on the same topics of the curriculum established by the Ministry of Education.
\end{abstract}

Keywords: brain, neuroeducational strategies, neuroscience, neurodidactics, teaching praxis 


\section{Introducción}

Entre 1990 y 2000 apareció la llamada década del cerebro (Barrios-Tao, 2016), donde la mayoría de los científicos se inclinaba más por el descubrimiento del funcionamiento del cerebro, ante cualquier actividad, siendo la más considerada en el campo educativo, ya que no se puede entender la educación si no se toma en cuenta cómo funciona el cerebro.

Existe una relación de las inteligencias múltiples, que son un modelo de entendimiento de las diferentes mentes que cada ser tiene; con la neurodidáctica, que es la disciplina que estudia el proceso de enseñanza-aprendizaje, basado en el desarrollo del cerebro; porque, parafraseando a Escobar (2015, p. 40), cada quien tiene su canal perceptual, estilos de aprendizaje VAK (neurolingüística) basado en el modelo de Bandler y Grinder (Hermosilla, 2020), que permite el trabajo conjunto y potencializador de enseñar por distintas vías al estudiantado, llegando así a que su proceso de enseñanza-aprendizaje sea de motivación propia y de manera significativa. Además, habría una mejora progresiva en la dinámica de las clases, incluyendo la iniciativa de que el estudiantado aprenda por sí mismo, con propios criterios, habilidades sociales y emocionales tanto para sí, como para con los demás.

Es necesario recalcar que cuando el equipo docente está en constante actualización, va desarrollando distintas destrezas que incluso puede compartirlas con sus estudiantes, generando una adaptación de las edades con las que se esté trabajando para fortalecer y desarrollar los conocimientos. Por ello es que los cursos de formación o capacitación hacia este equipo, inculcan nuevas maneras y/o estrategias que les permiten seguir adelante en su labor educativa. Para justificar lo mencionado, se toman los siguientes artículos de la LOEI (Ley Orgánica de la Educación Intercultural, 2017):

- Artículo 311: el nivel central de la autoridad educativa nacional, con el objeto de mejorar las competencias de los profesionales de la educación, certifica, diseña y ejecuta procesos de formación en ejercicio, atendiendo a las necesidades detectadas a partir de los procesos de evaluación (...).

- Artículo 313: la formación permanente de carácter remedial es obligatoria y se programa para ayudar a superar las limitaciones que tuviere el docente en aspectos específicos de su desempeño profesional.

Ahora bien, docentes de toda categoría deben realizar este tipo de actualizaciones, capacitaciones y mejoras pedagógicas que se detallan en la LOEI. Con ello cada clase será diferente de las comunes, habrá un ambiente de confianza, de conocimientos y de nuevas maneras de aprender procesual, experiencial y palpablemente de cada cerebro que esté bajo la mediación apropiada.

Finalmente, el objetivo 8 del Plan Nacional de Desarrollo 2017-2021 (Senplades, 2017), dispone: "fortalecer las capacidades y potencialidades de la ciudadanía"; y, dentro de la política 4.2, literal e), se dispone: "generar mecanismos pedagógicos, metodológicos y de enseñanza que promuevan la adecuada transición de los estudiantes a través los diferentes niveles de educación"; asimismo, en las políticas 4.4 y 4.5 se dispone: " 4.4 mejorar la calidad de la educación en todos sus niveles 
y modalidades, para la generación de conocimiento y la formación integral de personas creativas, solidarias, responsables, críticas, participativas y productivas, bajo los principios de igualdad, equidad social y territorialidad. 4.5. potenciar el rol de docentes y otros profesionales de la educación como actores clave en la construcción del buen vivir

En esa misma línea, se puede comprobar que al tener todo tipo de actualizaciones en el ámbito laboral-escolar, se va dando la oportunidad para el docente y el estudiantado, de que enseñe y aprenda integralmente bajo una guía adecuada y preparada.

En este sentido, el objetivo principal de la investigación es describir la experiencia de los docentes de EGB Elemental de la aplicación de la Neurodidáctica para mejorar la praxis de enseñanza y aprendizaje del subnivel.

Mientras que los específicos serían:

1. Caracterizar los elementos teóricos de la Neurodidáctica y su vinculación con las Inteligencias Múltiples.

2. Conocer los significados que otorgan los docentes dentro de su praxis educativa a las estrategias metodológicas para llevar a cabo el proceso de enseñanza y aprendizaje.

3. Analizar el valor que dan los docentes de EGB Elemental a las estrategias de la Neurodidáctica dentro de su praxis educativa.

4. Conocer cómo los docentes desarrollan los procesos de enseñanza y aprendizaje a través de la Neurodidáctica como herramienta para mejorar la praxis educativa.

\subsection{Marco teórico}

"El ser humano está dotado no solamente de habilidades cognitivas, de razón, sino también de habilidades emocionales, sociales, morales, físicas y espirituales; todas ellas provenientes del más noble órgano de su cuerpo: el cerebro" (Campos, 2010, p.3). De esta manera, al conocer básicamente el cerebro, permitirá que el ser humano tenga una gran flexibilidad para aprender creando variedad de sinapsis. Estas son las conexiones que se convierten en conexiones significativas cuando se ha tomado en cuenta la vía de aprendizaje más certera del educando. Considerando a Obando (2017), donde menciona a Gota y Cortés en su tesis "Neurociencia: herramienta para facilitar el aprendizaje" (2008), los profesionales de la educación que incorporen a su repertorio de destrezas docentes este nuevo conocimiento científico, sobre cómo el cerebro aprende, diseñarían de mejor manera sus planificaciones y junto con toda la comunidad educativa llegarían a un acuerdo de cómo aplicar el currículo.

Además, la neuroeducación es un medio idóneo para realizar activamente los procesos de enseñanza aprendizajen debido a la oportunidad de estimular el cerebro en donde haya la trilogía de teoría, práctica y reflexión. Es a través del educador, donde con las actividades previamente planificadas, se desarrollará la parte crítica y reflexiva del estudiante. 
Relacionando la neurodidáctica con las inteligencias múltiples, Gardner, mencionado en Obando (2017), dice que estas inteligencias no se encuentran aisladas, sino que se combinan para llegar a tener cierto conocimiento. De este modo, el docente debe entender su trabajo desde la teoría de las inteligencias múltiples, para evaluar los intereses y capacidades de los estudiantes, incrementando la autoestima y el entusiasmo de trabajo en equipo.

Por tanto, Campos (2010) hace alusión a que "el panorama que se aprecia en las aulas actualmente acaba siendo el de una práctica pedagógica híbrida, resultante de tantas corrientes y líneas, muchas de ellas ya sobrepasadas, y que no corresponden al perfil de alumno que frecuenta la escuela del Siglo XXI" (p.4).

Como educadores, se debe ir más allá de lo que comúnmente se lo ha realizado por varios años, la generación de estudiantes que están pasando por la educación actual desea más de lo que se les está dando, hay que saber potenciar el ambiente para que la clase surja adecuadamente con la praxis docente juntamente con la del educando.

Añadiendo a lo que antecede, para Aristizábal (2015), el proceso de aprendizaje, visto desde el aspecto biológico, "se encuentra que el aprendizaje es una función fundamental de las neuronas que están compuestas por un cuerpo celular, las dendritas y los axones" (p. 8). He aquí la importancia de como docentes, comprender el funcionamiento básico del cerebro. Finalmente, surgen algunos principios de la neuroeducación (Obando, 2017, p. 16), en los cuales podemos basarnos para una activación oportuna de los neurotransmisores en el aula.

- Cada cerebro es único y organizado; aunque todos los seres humanos tienen los mismos patrones en la organización cerebral, cada quien percibe los estímulos de diferente manera, ya que dependen de factores elementales como su contexto social y sus propias experiencias. Así, el docente respetará cada ritmo y estilo de aprendizaje de sus estudiantes, sea kinestésico, visual, lingüístico o auditivo, generando el aprendizaje significativo y duradero.

- El cerebro es un sistema complejo y dinámico que cambia diariamente con la experiencia; "sistema complejo debido a que en él se origina la sinapsis proceso cerebral a través del cual se produce el aprendizaje" (Obando, 2017) y para que se sigan dando estos procesos sinápticos es necesario la repetición de actividades. Se habla de dinámico porque como se ha mencionado en el anterior principio, se necesita de las experiencias de interacción social para que el estudiante llegue a crear significativamente su aprendizaje.

- La búsqueda del significado es innata en la naturaleza humana; desde que el ser humano nace es instintivo y sobrevive a ese medio, por ello es que somos curiosos por naturaleza y gracias a esta cualidad podemos encontrar el porqué de las cosas.

- El aprendizaje, en parte, se basa en la capacidad del cerebro para autocorregirse; el aprendizaje se da por aciertos y errores, y el cerebro es capaz de corregirlo. Por ello es que en las clases se debe aceptar el error y retroalimentar a los estudiantes, no viéndolos como una amenaza, sino como un medio para aprender y corregir. El cerebro busca la novedad y donde tome lugar los contextos naturales; el cerebro busca ese algo novedoso que le llame 
la atención para aprender de mejor manera. Además, la memoria funciona mejor cuando se enseña con contexto real del estudiante, asemejando sus vivencias, y el docente puede utilizar diversos recursos que permitan desarrollar este proceso de E-A.

- Las emociones son críticas para detectar patrones para la toma de decisiones y aprender, mejorando con retos y desafíos, más no con amenazas; la relación entre emociónaprendizaje es fundamental en la neurodidáctica, ya que el ser humano es muy emocional, y dependiendo de ello tendrá éxito o fracaso en su vida de manera general. Salazar (2005) explica que "cuando los estudiantes están emocionalmente comprometidos con el aprendizaje, ciertos neurotransmisores en el cerebro envían señales al hipocampo, estructura vital del cerebro que incluye la memoria, para estampar estos eventos con gran intensidad" (p.12).

- El feedback es importante para el aprendizaje; con ello se logra que un conocimiento quede fijo en nuestro cerebro, si no se refuerza la información pasa a ser desechada.

\section{Metodología}

La metodología de este trabajo se basa en el enfoque cualitativo (Hernández et al., 2014), el cual ha permitido que la investigación se desarrolle de manera inductiva, teniendo como fuentes teóricas trabajos de Izaguirre (2017) y Obando (2017) sobre la neuroeducación en el aula, y cómo mejorar la enseñanza y aprendizaje con estrategias constructivistas, para así, junto con las prácticas de los docentes de EGB Elemental, llegar a resultados propicios de cada nivel de instrucción. Éste último se logra gracias al método hermenéutico, ya que permite llegar a las interpretaciones de la vida de los educadores, informantes clave, a través de su experiencia narrada y hecha texto.

El escenario de investigación fue en una Unidad Educativa del norte de Quito - Ecuador, en donde el equipo docente implementó hace cuatro años el método de las inteligencias múltiples en sus aulas, para enseñar de manera integral a sus estudiantes.

La muestra fue representada por los cuatro docentes integrales de EGB Elemental y dirigido al estudiantado del mismo subnivel, que figuran a segundo, tercero y cuarto de básica. Éstos cumplieron con un perfil para llevar a cabo este trabajo, teniendo como características: conocimiento, capacitaciones y/o alguna relación en el campo de investigación, como características con respecto a la neuroeducación y/o neurodidáctica, significados, relación con la educación, apoyo escolar, estrategias metodológicas, el funcionamiento del cerebro dentro del aprendizaje.

Las técnicas utilizadas fueron de observación y grupo focal (Mella, 2000), cuyos instrumentos se plasmaron en tablas, asignando las categorías de: unidades de análisis y elementos a observar, ambas identificadas en el marco teórico; de esta manera resultó fácil desarrollar las anotaciones y desde diferentes perspectivas de interacción.

\section{Resultados}


Como se lo menciona en el párrafo anterior, la investigación fue desarrollada en base a dos instrumentos que permitieron conocer dos aspectos por parte de los informantes clave.

Por cada matriz se realiza una síntesis de la información obtenida, por lo tanto, los resultados se observarán después de cada tabla presentada a continuación.

A través de esta visión general de las observaciones realizadas, se describe que la combinación del funcionamiento del cerebro con la pedagogía es esencial para el desarrollo de un proceso de enseñanza-aprendizaje creativo y de interés en cada estudiante.

Además, se asegura que los docentes deben estar en constante actualización e investigación para llegar a ser innovadores y precursores de una educación diferente. Y, para ello, los principios de neuroeducación son y serán necesarios para tomarlos en cuenta en todo momento de la clase, considerando las sinapsis adecuadas de conocimiento, con las estrategias y recursos neuroeducativos, correspondientes para cada uno de los actores de la educación, docentes y discentes.

Tabla 1.

Observación

\begin{tabular}{|c|c|c|}
\hline Unidad de Análisis & Elementos a observar & Observaciones \\
\hline 1) Qué es - praxis & $\begin{array}{l}\text { Combinación del } \\
\text { funcionamiento del cerebro } \\
\text { con la pedagogía. } \\
\text { Proceso educativo } \\
\text { (reflexión acción) } \\
\text { Proceso enseñanza- } \\
\text { aprendizaje innovador y } \\
\text { creativo }\end{array}$ & $\begin{array}{l}\text { a) De las observaciones de clases se analiza que sí es } \\
\text { preciso tomar en cuenta la combinación del funcionamiento } \\
\text { del cerebro con la pedagogía en cada clase que se realiza, } \\
\text { porque cada persona tiene su vía de aprendizaje. En este } \\
\text { caso, el mediador genera el ambiente apropiado para } \\
\text { llegar a sus estudiantes, haciéndoles que abran su mente } \\
\text { integralmente y obtener ese aprendizaje significativo. } \\
\text { b) De manera general, se identifica que la praxis docente } \\
\text { no ha sido un proceso totalmente consciente, pero si tienen } \\
\text { en cuenta que, al poder darse esa oportunidad de reflexión, } \\
\text { darán a notar a sus estudiantes que, si son conscientes de lo } \\
\text { que hacen, podrán generar procesos de reflexión para dar el } \\
\text { paso a diferentes acciones y ver la que mejor convenga. Esto } \\
\text { se da como se lo ha mencionado anteriormente, con una guía } \\
\text { adecuada del docente. } \\
\text { c) El proceso de enseñanza-aprendizaje va siendo } \\
\text { innovador, no solo en un día o semana, se requiere de } \\
\text { tiempo, constancia y dedicación para reabrir los campos de } \\
\text { investigación del docente. Una vez que esté consciente de los } \\
\text { cambios que realizará, está listo para hacer su innovación, } \\
\text { y no únicamente con recursos tecnológicos, porque el } \\
\text { ser innovador y creativo es buscar medios diversos de } \\
\text { enseñanza-aprendizaje para llegar, con motivación e interés, } \\
\text { a cada estudiante. Por parte del docente, este puede ser } \\
\text { innovador y/o creativo no exactamente en todas las clases, } \\
\text { pero sí puede ser una buena opción para al menos dos veces } \\
\text { a la semana realizarlo. }\end{array}$ \\
\hline
\end{tabular}


2) Estrategias neuroeducativas
Proyectos por equipo

Juego

Juegos mentales

Pausas activas

(movimientos)

Emociones

Artes y música

Recursos tecnológicos a) De manera general, los proyectos por equipo son factibles para la neurodidáctica, porque los estudiantes van conociendo a sus compañeros, definiendo roles que generan una interacción social amistosa con el grupo. Cada semana, los estudiantes de segundo EGB estaban sentados en diferentes puestos y con nuevos compañeros de trabajo, en cambio, en cuarto EGB se les ubicaba en grupos de cuatro o cinco estudiantes en una mesa, para trabajar los diferentes proyectos establecidos mensualmente.

b) En general, el juego fue más presente es en los años de segundo EGB, donde las docentes no necesitan de mucho material para hacer y es una manera lúdica de enseñarles las letras para facilitar su escritura. En cambio, para los cuartos años, no se aplicó en las clases observadas esta otra forma de aprender. Sin embargo, el juego puede ser un medio fundamental para aprender conocimientos no necesariamente en niveles básicos, sino también superiores.

c) La presencia de juegos mentales fueron enraizadas con el tema que estaban viendo en cierto proyecto, por ejemplo, el tema era sobre los huesos y los profesores realizaban juegos preliminares para que los estudiantes se introduzcan al tema de aprendizaje.

d) Las pausas activas eran relevantes para los cuatro informantes observados, puesto que se sentía que cuando avanzaban con la clase iban perdiendo conexión con el mundo y se distraían. Por ello es que docentes aplicaban estas pausas activas, para reactivarles y que su cuerpo se sienta relajado para continuar con las actividades.

e) La estrategia de trabajar las emociones no fue muy aplicada por todas las informantes; sin embargo, las docentes de cuarto año demostraron que enseñar a autorregular las emociones se lo puede hacer en momentos precisos, donde se vea que los estudiantes muestren diferente su estado de ánimo, haciendo actividades sencillas y que duran poco, pero a la vez relaja un tiempo moderado.

f) La música se notaba que frecuentaba cada clase, pese a esto, presenciándolas no estuvo explícitamente dada por algún tema de clase o por parte de planificación, además de que puede ser un medio para despedirse.

g) Los recursos tecnológicos que tienen los salones de clase son laptops y un proyector. Sin embargo, no fue muy necesario utilizar, más el proyector, para dar clases, ya que se utilizaban otros materiales. Por otro lado, las docentes si utilizaban la laptop para poder buscar más información sobre cierto tema a dar, o para ver correos institucionales o pasar notas. 
3) Importancia estilos de aprendizaje
Relación afectiva y respetuosa.

Actividades visuales, auditivas, kinestésicas y/o sensoriales.

Flexibilidad con los estudiantes al realizar una tarea. a) y c) Se han unido estos dos literales porque a través de la investigación se notó la conexión que hay en estas dos para que el aprendizaje se lleve a cabo. Cuando hay una buena relación de respeto, confianza y consideración, los estudiantes sienten seguridad para realizar sus actividades, como decía una informante, arriesgarse para hacer o decir algo, siempre pensando en que cada niño es mundo y tiene su manera de decir o hacer algo. A pesar de que es un número grande de estudiantes (26), las docentes intentan brindar sus conocimientos y retroalimentarlos de manera personal, sea cuando realizan un trabajo grupal o cuando revisa las actividades y/o tareas.

b) Las actividades observadas en su mayoría eran visuales y auditivas, pero así mismo se presenciaron actividades kinestésicas (de movimiento corporal) y una que otra sensorial. De esta manera se demuestra que las actividades deben ser pensada de manera integral, para que así el estudiante pruebe diferentes caminos para obtener su propio conocimiento.
4) Inteligencias múltiples

Desenvolvimiento del
estudiante en cierto
entorno o ante cierta
situación.
Combinación de
inteligencias para llegar a
tener cierto conocimiento.

Desenvolvimiento del

a) Se determina que las docentes permitían que sus estudiantes se expresen ante ciertas situaciones (académicas y/o personales); les daba la oportunidad de reflexionar sobre sus respuestas, el docente no les decía que estaba mal, más bien utilizaban la frase: y si intentas de esta otra manera... así daban confianza permitiendo que se desenvuelvan naturalmente en cualquier entorno.

b) En esta institución se trabaja con la paleta de inteligencias múltiples para ejercer el proceso de enseñanza-aprendizaje. De esta manera, los docentes planifican y desarrollan los temas basándote en las 8 inteligencias, aplicando cada día o cada dos días una diferente. Esto permite que los estudiantes lleguen a captar su atención y mejorar sus conocimientos por medio de la vía (inteligencia) que sea la suya. Las inteligencias que se observaron fueron: musical, kinestésica, inter e intrapersonal, lógico-matemático, lingüística y visoespacial. 
5) Participación activa a) y b) Se han conectado estos dos elementos de observación debido a que se ha presenciado que la capacidad cognitiva, emocional y de interacción social tienen un gran peso cuando se va aprendiendo algo nuevo. La reflexión individual del estudiante es muy valiosa, y las docentes permitían que ellos mismos se den cuenta de lo que estaban haciendo, generando significancia en lo aprendido diariamente. En ciertos casos no solo la auto-reflexión se daba a conocer, también la co-reflexión que incluso sorprendía a las propias educadoras de lo que eran capaces sus estudiantes cuando su cerebro, cuerpo y alma trabajan juntos.

c) Las técnicas neuroeducativas utilizadas por las docentes fueron similares, debido al sistema que EGB Elemental maneja; estas fueron: códigos $(0,1,2,3)$ para fijar la tonalidad de la voz en cierto trabajo; canciones con poco movimiento corporal, con los fines de reconectarse en la clase o pausas activas; contabilizar el tiempo de trabajo con palitos en pizarra o alarma bomba proyectada; posiciones diferentes de mesas; los docentes dicen: alce la mano quien me escucha y me mira; DELES (Dibuja, Escribe, Lee En Silencio) para quien termina la actividad asignada. 
6) Principios

Neuroeducación - praxis
Cada cerebro es único y organizado que cambia conforme su experiencia La búsqueda del significado es innata en la naturaleza humana Aprendizaje en parte se basa en la capacidad del cerebro para autocorregirse Cerebro busca la novedad y donde tome lugar los contextos naturales Emociones son críticas para detectar patrones, tomar decisiones y aprender (no amenazas) Feedback para el aprendizaje a) Lo que se ha presenciado de manera general es que efectivamente, hasta las docentes tienen un cerebro único y organizado que para aprender o enseñar algo lo hacen en base la vida real, rutinas o experiencias que cada uno ha tenido. Por esta razón es que para algunos estudiantes se les hacía fácil hacer una actividad, mientras que a otros les tomaba más tiempo comprender para poder llevarlo a cabo. b) Se determina que los estudiantes de EGB Elemental son curiosos en el buen sentido de la palabra, permitiéndoles encontrar el porqué de las cosas por ellos mismos, preguntando a los compañeros o también a sus docentes. Ellas les hacían reflexionar sobre sus actos, cuando ocurría algún inconveniente con un compañero, o incluso para dar a conocer sus puntos de vista.

c) El aprendizaje percibido durante estos siete días ha sido de aciertos y errores, donde las docentes les han encaminado para que lleguen a su propia reflexión del error. Por otro lado, hay estudiantes que se dan cuenta de su error inmediatamente, y fue sorprendente ver su expresión de sentirse equivocado, pero a la vez de haberse dado cuenta él o ella solo(a) para poder hacerlo mejor. Si es posible que nos autocorrijamos.

d) Se estipula, de todas las clases observadas, que las docentes dieron la mayor parte del contenido en base a contextos reales de los estudiantes. Esto permitía que haya una mayor participación y que la clase se desarrolle con facilidad. También, se desarrollaron actividades novedosas donde los estudiantes estaban atentos a la siguiente indicación de la docente para seguir avanzando con su aprendizaje.

e) Definitivamente las emociones son cruciales para el desenvolvimiento oportuno y adecuado de una persona en cualquier entorno. En el caso de los estudiantes, al inicio de la jornada ellos colocaban en un "emocionómetro" su estado de ánimo, esto permitía conocer a los docentes cómo poder trabajar o si no se avanzaba era porque algo pasó en casa o con sus amigos. Si estaban irritados o cansados, su nivel de atención bajaba y no ponía atención, por lo que el trabajo se quedaba incompleto, afectándolo en todo aspecto de su ser. En cambio, si estaban felices o entusiasmados, las actividades fluían mejor, gracias a las retroalimentaciones que como docentes se debe comentar.

f) Los docentes si aportaban reiteradas retroalimentaciones para un mejor entendimiento de las actividades solicitadas. En ciertas ocasiones, los estudiantes se quedaban callados y pedían ayuda al grupo; sin embargo, la docente se paseaba por el aula para asesorarse de que estuviera todo bien y entendido de manera individual y grupal. Así es como se permite que el proceso reflexivo de E-A sea el adecuado para todos en el aula. 


\begin{tabular}{ll}
\hline 7) Correlación entre IM y & Tema abordado en clase \\
Neurodidáctica & contextualizado con el \\
diario vivir del alumno & Capacidad de \\
razonamiento & Material lúdico, didáctico, \\
novedoso y/o tecnológico & Motivación hacia el \\
aprendizaje, captación \\
de su atención y trabajo \\
en las memorias más \\
significativas
\end{tabular}

a) Como se mencionó anteriormente, las docentes abordaban los temas de aprendizaje hacia contextos en los que la mayoría de estudiantes había presenciado en algún momento porque había una buena participación activa. Además de que eran temas conocidos por anteriores años en la escuela, por contexto familiar o entre compañeros hablaron en otra ocasión, logrando una conexión inmediata con el tema a tratar.

b) y d) Se ha conectado estos dos elementos observados puesto que con la capacidad de razonamiento que las docentes potenciaban en sus estudiantes, ellos se sentían motivados por seguir aprendiendo nuevos temas. Los razonamientos en algunos casos eran individuales y en otros colectivos, donde es posible aplicar técnicas de juego para que poco a poco lleguen a convertir sus dudas en aprendizajes y experiencias propias de sí. Finalmente, es posible que los docentes relacionen contenidos vistos en otras materias, para conjugarlos con los que se están dando en ese momento, ya que habrá una mejor conexión y captación del tema por parte de todos los estudiantes.

c) Los materiales utilizados permiten llamar la atención del estudiantado, por lo que como docentes se debe aprovechar y crearlos. No es necesario solo tener materiales tecnológicos, se pueden utilizar de todo tipo, pero lo importante es que genere la participación activa y consciente de cada alumno.

De acuerdo con la Tabla 1 se sintetiza que efectivamente el uso del funcionamiento del cerebro con el aprendizaje si se puede dar, siempre que el equipo docente esté dispuesto a realizarlo para que el proceso de enseñanza-aprendizaje sea óptimo, flexible, reflexivo y sobre todo creativo, para cada tema a aprender en clase.

Con respecto a la unidad de análisis de estrategias neuroeducativas, las docentes utilizan con mayor frecuencia proyectos por equipo, para potenciar las relaciones intra e interpersonales; emociones, enseñarles a regularse cuando algo ha pasado para actuar de buena manera; y la música, medio de comunicación para la relajación, disciplina e incluso reconexión con las clases.

La siguiente unidad de análisis, importancia de los estilos de aprendizaje, se determina en los cuatro informantes que crean un ambiente propicio y de convivencia para aprender, porque genera confianza y seguridad en el grupo. Por ello es que las planificaciones curriculares deberían ser tomadas en cuenta con los estudiantes, también para poder generar clases interesantes y llamativas para ellos.

Se han relacionado las unidades de análisis inteligencias múltiples y correlación de I.M. con la neurodidáctica, porque al trabajar con la paleta de I.M. se están enfocando en la manera de aprender de cada educando; es decir, en su vía de aprendizaje más desarrollada. En las aulas se observó que cada cierto tiempo trabajan cada inteligencia de manera separada, lo que permite que el estudiantado pruebe su aprendizaje desde diferentes aspectos. 
La participación activa es otra unidad, de la cual se identifica que para que todos los estudiantes estén atentos y motivados hacia el aprendizaje, se necesita de un ambiente propicio y, también, de un docente que quiera ser creativo e innovador para salir de lo ortodoxo y animarlos a seguir aprendiendo.

Con respecto a los principios de la neuroeducación se concluye que el cerebro está fuertemente ligado con el aprendizaje, no solo de la escuela, sino también fuera de ella, lo que amplía el campo de aprendizaje con más opiniones e ideas que compartir, para generar nuevos conocimientos en base a una reflexión adecuada.

Mediante la Tabla 2 se dará a conocer las ideas relevantes que los cuatro informantes expusieron durante el grupo focal. De ello se ha determinado que la parte teórica comentada, se vincula con lo que se ha observado durante las clases. Una de ellas es pensando en los estilos de aprendizaje que cada educando tiene. En cuanto a los principios de neuroeducación, mencionan en que se debe utilizar en todo momento y siempre priorizando la reflexión y el ser consiente de los actos y actividades que están realizando. También se comenta que las actualizaciones e innovaciones como docente son importantes para llegar hacia los estudiantes en su desarrollo humano, cognitivo e integral. Finalmente se destaca que los docentes no deben ser los únicos en conocer sobre el funcionamiento básico del cerebro, cualquier persona involucrada con estudiantes debe conocer el desarrollo del mismo y llegar eficazmente al nuevo conocimiento.

Aprendizaje que cada educando tiene. En cuanto a los principios de neuroeducación, mencionan que se debe utilizar en todo momento y siempre priorizando la reflexión y el ser consiente de los actos y actividades que están realizando. También se comenta que las actualizaciones e innovaciones como docente son importantes para llegar hacia los estudiantes en su desarrollo humano, cognitivo e integral. Finalmente se destaca que los docentes no deben ser los únicos en conocer sobre el funcionamiento básico del cerebro, cualquier persona involucrada con estudiantes debe conocer el desarrollo del mismo y llegar eficazmente al nuevo conocimiento. 
Tabla 2.

Grupo focal

\begin{tabular}{lll}
\hline Unidad de Análisis & Pregunta / Subpregunta & Respuesta conclusiva \\
\hline 1) Qué es - praxis & 1. Con respecto & 1) La parte de la neurociencia en la educación ha \\
a los términos de & permitido que esta nueva herramienta neuroeducativa \\
neuroeducación y & se pueda utilizar en cualquier momento, siempre \\
neurodidáctica, ¿qué & comenzando con el observar y conocer a los estudiantes, \\
aportes nos puede & es decir, en el contexto que les rodea día a día, porque \\
comentar acerca de & van a ser quienes estén mucho tiempo frente a uno con \\
esta nueva herramienta & una determinada necesidad. \\
pedagógica? & Además, los estudiantes ya vienen con conocimientos \\
& previos desde casa, por lo que los docentes no hacen \\
2. ¿Qué entiende usted por & actividades por ellos, les permiten ir desarrollando más \\
praxis docente? & el conocimiento y abriendo todas esas ramas para que \\
& el mismo estudiante asimile la información y haga propio \\
& su aprendizaje. Para ello depende mucho de la formación \\
& que cada docente tenga. \\
& 2) La praxis docente es un método más humano y \\
& consiente en cómo uno se enfoca en el aula para dar \\
& las clases, siempre con una planificación, permitiéndola \\
& ser flexible para llevarla a cabo con todas las dudas y \\
& sugerencias de los estudiantes. Además, hay que pensar \\
& y tomarlo muy en cuenta que cada docente viene con una \\
& programación diferente, por lo que hay que adaptarse y \\
& evolucionar junto con los estudiantes.
\end{tabular}

2) Estrategias

neuroeducativas de

Participación activa
3. ¿De qué manera ha influenciado la aplicación de estrategias neuroeducativas en su praxis docente?

\section{4. ¿Alguna experiencia} neuroeducativa que le ha sido de mucha utilidad para controlar la disciplina de su aula?
3) Es muy importante conocer el contexto de cada estudiante, porque ellos son personas que se están formando, que están desarrollando su cerebro y que cada instante necesitan nutrirse de más para satisfacer esa felicidad de conocer más cada día. Cada quien tiene un mundo y es muy interesante llegar a conocer al menos una parte de él permitiéndole crecer en su integridad. 4) La estrategia aplicada y más útil es la música, ya que ella permite momentos de relajación, acompañados de respiraciones y movimientos, para que el estudiante se conecte nuevamente con las actividades que se están realizando. Hay que saber en qué momentos aplicarla, porque no siempre va a funcionar y es mejor pensar en todos los estudiantes para que la música no sea un instrumento de distracción, más bien de aprendizaje y concentración. La música, vista no solo desde las canciones que se reproducen en CDs o computadoras, también se hace música con el cuerpo y manos y los pies, donde se aplaude o se zapatea para generar alguna vinculación con el aprendizaje. De alguna manera ellos se conectan y toman a la canción como un momento de término de alguna actividad en clase, siempre tomando en cuenta los acuerdos y el bienestar de todos. 


3) Importancia estilos de
aprendizaje
4) Inteligencias múltiples
7) Vinculación IM y
Neurodidáctica
neuroeducación - praxis
Debido a los avances tecnológicos, los científicos siguen acentuando datos sobre el funcionamiento del cerebro durante los momentos de aprendizaje. Según estos antecedes, 5. ¿qué opinión merece por usted, ser recalcada con respecto al conocimiento básico del cerebro durante el proceso de enseñanza- aprendizaje?

6. ¿Conoce usted la
vinculación que existe
entre la neurodidáctica y
las inteligencias múltiples?
Explique brevemente.

5) Todas las personas que estén en contacto con los niños, niñas o adolescentes, e incluso adultos y mayores, deben tener conocimiento básico del desarrollo del cerebro, su funcionamiento es primordial, y siempre partiendo, en el proceso de enseñanza-aprendizaje, de las experiencias para que sea más propio de sí los conocimientos que vaya adquiriendo. Se sugiere la gimnasia cerebral, donde niños/as a partir de los tres años pueden asistir para igualar los dos hemisferios y poner en práctica muchas más actividades, siempre pensando en el nivel cognitivo, social y cultural de la persona. La tecnología hoy en día es importante y habrá que saber utilizarla de la mejor manera, desde los docentes, para que el conocimiento del cerebro sea elemental en todos. 6) La vinculación que tienen las inteligencias múltiples con la neurodidáctica es bien cercana y van de la mano durante el proceso de enseñanza-aprendizaje de cada educando, porque el cerebro está en constante actividad y se desarrolla más gracias a la habilidad más potenciada. He ahí la importancia de que como docentes se los enganche hacia el aprendizaje con sus propios intereses.
7. ¿Cuál considera usted que sea la importancia de aplicar la neurodidáctica como herramienta pedagógica dentro del salón de clase? Y ¿por qué?

\begin{abstract}
7) El tener la vocación permite desarrollar de mejor manera la parte perceptiva en el docente, ya que se va conociendo más a esa persona que está en frente y va comentando u opinando cómo realizar alguna actividad, es primordial tomarlos en cuenta porque va saliendo de ellos la vía más rápida para llegar al aprendizaje, siempre manteniendo los parámetros.

Además, al realizar actividades donde se utilice materiales de todo un poco o distintas actividades, quiere decir que el niño siempre aprende por sus sentidos, ya que cuando hay un buen estímulo, éste llegará directamente a su cerebro con mayor captación.
\end{abstract}

Con referencia a las preguntas 1 y 2 de la unidad de análisis, se determina que la neurociencia en la educación es una herramienta fundamental para hacer de las ortodoxas clases, más amenas y divertidas, en el ámbito de que cada estudiante es libre de aprender de acuerdo a su manera de ser, con la guía que siempre los educadores se les proporciona para llegar al objetivo común, a ese conocimiento nuevo. Por tal motivo es que la praxis de los docentes debe ser la primera que se reflexione para contagiar del mismo entusiasmo y actitud a los estudiantes, y que puedan accionar, pensando bien, sus actividades.

En la siguiente pregunta de la unidad de análisis planteada como estrategias neuroeducativas, se describe que la mejor estrategia para comenzar con la intervención de neuroeducación es conocer el contexto de cada uno de los estudiantes, porque son un mundo único con necesidad de aprender y nutrir su cerebro por medio de nuevos conocimientos y de la interacción social.

Con respecto a la pregunta 4, se tuvo como respuesta que para que se dé con mayor fluidez la participación de los estudiantes es necesario utilizar diferentes estrategias que fomenten la generación de ideas y/u opiniones. Por ello es que la estrategia más utilizada por parte de todas, según lo mencionado también, es la música, teniendo canciones de CDs o videos, pero con mucha 
más frecuencia el uso del cuerpo para que haya conectividad con los temas de clase, además de momentos de relajación.

En cuanto a la pregunta 5 de las unidades de análisis de importancia en estilos de aprendizaje, inteligencias múltiples y vinculación IM y neurodidáctica, se identifica que todas las personas que estén en contacto con los niños/as, adolescentes y/o adultos en el ámbito educativo, es necesario que el educador tenga conocimiento básico del desarrollo del cerebro, y más si es con la ayuda de la tecnología, el conocer su funcionamiento será más sencillo para aplicarlo con los estudiantes en su mejor vía de aprendizaje.

De la pregunta 6 se obtiene que las I.M. y la neurodidáctica tienen una estrecha relación durante el proceso de enseñanza-aprendizaje de cada educando, porque el cerebro, al ser plástico, se va moldeando y desarrollando según la personalidad de cada niño, y además potencia una inteligencia más que otra.

Con referencia a la pregunta 7 de la unidad de análisis principios de neuroeducación, se determina que los educadores tienen más desarrollada esa parte perceptiva de saber lo que pasa con los educandos, ya que ello permitirá un mejor desenvolvimiento de las clases incentivando el diálogo entre todos.

Mediante las dos técnicas de investigación realizadas, observación y grupo focal, se fue interpretando cada unidad de análisis desde el punto de vista individual y colectivo de las docentes. A partir de ello, y las síntesis obtenidas, se ha realizado una triangulación (Tabla 3), que es la técnica de análisis de contenido y conclusivo de todas las matrices anteriormente detalladas. 
Tabla 3.

Tabla de triangulación

\begin{tabular}{|c|c|}
\hline & \\
\hline $\begin{array}{l}\text { ape se reallza. } \\
\text { ue este } \\
\text { tes, sino } \\
\text { enerando } \\
\text { a } \\
\text { studiantes } \\
\text { liendo roles } \\
\text { osa con el }\end{array}$ & $\begin{array}{l}\text { Ir y conocer a los estudiantes, dentro del contexto } \\
\text { rodea día a día. } \\
\text { is docente es un método más humano y consiente } \\
\text { uno se enfoca en el aula para dar las clases, } \\
\text { con una planificación, permitiendo que sea } \\
\text { dien tiene su mundo y es importante conocer el } \\
\text { y las vías de aprendizaje más desarrolladas del } \\
\text { tado, ya que su aprendizaje será para todos los } \\
\text { de la vida. }\end{array}$ \\
\hline
\end{tabular}
aprendizaje significativo, tomando en cuenta hechos novedosos y de interés para los estudiantes.

\section{Conclusiones}

A través de la triangulación, según mencionan Okuda y Gómez (2005), "se cree que las debilidades de cada estrategia en particular no sobreponen con las de las otras y que en cambio sus fortalezas si se suman (...) ofrece la alternativa de visualizar un problema desde diferentes ángulos". Tal es así, que se analizaron las conclusiones que se interpretaron de la observación, del grupo focal y análisis de la investigación.

La aplicación de la nueva herramienta pedagógica, vista desde el ámbito de la neurociencia, es de suma utilidad, de tal manera que uno como docente es capaz de llegar directamente al cerebro del estudiante para que el conocimiento que llegue a tener sea significativo, y esto se logra por conocer desde el contexto que le rodea al educando, hasta la inteligencia, mayormente desarrollada de cada uno de ellos. Siempre se deben buscar estrategias para que las clases sean novedosas y de interés, no solo dictar y dictar, con recursos neuroeducativos se puede llegar a una participación activa y un clima apropiado de aprendizaje, adaptado para el cerebro de cada estudiante. 
A través de la última tabla se detalla a manera de conclusión que las docentes de EGB Elemental tienen conocimiento general sobre la neurodidáctica y/o neuroeducación, lo cual permite recalcar que esta nueva herramienta pedagógica está a la vanguardia de la educación, porque está pensada en cada habilidad del educando. Tal es así que da la oportunidad de trabajar los mismos temas de la malla curricular que el Ministerio de Educación detalla, con la única diferencia que el equipo docente debe ser capaz de arriesgarse a probar nuevas estrategias que hagan del estudiante un ser crítico, pensador y participativo, ante cierta situación.

Hay que mencionar además que se estableció que la relación existente entre las inteligencias múltiples y la neurodidáctica sí está vinculada, van de la mano durante el proceso de enseñanzaaprendizaje, puesto que cada niño, niña, adolescente, joven, adulto e incluso adulto mayor, tiene su canal perceptual (VAK) y ritmo para aprender. El o la docente es el guía, el mediador que está a lado de cada mundo, fomentando en cada oportunidad su funcionamiento cerebral ante el conocimiento. Finalmente, las estrategias metodológicas para el desarrollo de clases y/o reconexión para abordar temas durante varias horas de clase, se concluye, con mayor garantía, que sí es posible realizar la neurodidáctica en el aula, siendo una herramienta de mejora. 


\section{Referencias}

Asamblea Nacional del Ecuador. (2017). Ley Orgánica de la Educación Intercultural. https://educacion.gob.ec/ wp-content/uploads/downloads/2017/05/Ley-Organica-Educacion-Intercultural-Codificado.pdf

Aristizábal, A. (2015). Avances de la Neuroeducación y aportes en el proceso de enseñanza aprendizaje en la labor docente. [Tesis de Grado, Universidad Militar Nueva Granada]. Repository Unimilitar http://hdl. handle.net/10654/6186

Barrios-Tao, H. (2016). Neurociencias, educación y entorno sociocultural. Educación y Educadores, 19(3), 395415. https://doi.org/10.5294/edu.2016.19.3.5

Campos, A. (2010). Neuroeducación: uniendo las neurociencias y la educación en la búsqueda del desarrollo humano.La Educ@ción, (143) 1-14. http://www.educoea.org/portal/La Educacion Digital/laeducacion 143/ articles/neuroeducacion.pdf

Escobar, V. (2015). Elaboración de archivos de audio con fines académicos en la Facultad de Psicología de la PUCE-Q al año 2014. [Tesis Maestría, Pontificia Universidad Católica del Ecuador] Repositorio PUCE http://repositorio.puce.edu.ec/handle/22000/10037

Hermosilla, K. (05 de mayo de 2020). Modelo de Bandler y Grinder. ABC. https://www.abc.com.py/edicion-impresa/suplementos/escolar/2020/05/05/modelo-de-bandler-y-grinder

Hernández Sampieri R., Fernández Collado C. \& Baptista L. (2010). Metodología de la Investigación. Editorial McGraw Hill.

Izaguirre, M. (2017). Neuroproceso de la enseñanza y del aprendizaje: metodología de la aplicación de la neurociencia en la educación. Alfaomega

Mella, O. (2000). Grupos focales (“Focus groups”). Técnica de investigación cualitativa. Documento de trabajo, (3).

Obando, A. (2017). Neuroeducación en el proceso de enseñanza y aprendizaje del idioma inglés, en estudiantes de octavo año de Educación General Básica, de la Unidad Educativa "Liceo Policial”, D.M. Quito, período 2016. [Tesis de grado, Universidad Central del Ecuador] Repositorio UCE. http://www.dspace.uce.edu.ec/ handle/25000/13155

Okuda Benavides, M. \& Gómez Restrepo, C. (2005). Métodos en investigación cualitativa: triangulación. Revista Colombiana de Psiquiatría, 34(1), 118-124. http://www.scielo.org.co/pdf/rcp/v34n1/v34n1a08.pdf

Salazar, S. (2005). El aporte de la neurociencia para la formación docente. Revista Electrónica "Actualidades Investigativas en Educación", 5(1) https://www.redalyc.org/pdf/447/44750102.pdf 
Secretaría Nacional de Planificación y Desarrollo. (2017). Plan Nacional de Desarrollo 2017-2021-Toda una Vida. Senplades. https://www.planificacion.gob.ec/wp-content/uploads/downloads/2017/10/PNBV-26OCT-FINAL OK.compressed1.pdf 
Copyright (c) 2022 Carolina Estefanía Saquicela Richards

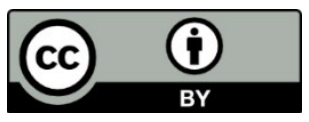

Este texto está protegido bajo una licencia internacional Creative Commons 4.0.

Usted es libre para Compartir-copiar y redistribuir el material en cualquier medio o formato - y Adaptar el documento - remezclar, transformar y crear a partir del material-para cualquier propósito, incluso para fines comerciales, siempre que cumpla las condiciones de Atribución. Usted debe dar crédito a la obra original de manera adecuada, proporcionar un enlace a la licencia, e indicar si se han realizado cambios. Puede hacerlo en cualquier forma razonable, pero no de forma tal que sugiera que tiene el apoyo del licenciante o lo recibe por el uso que hace de la obra.

$\underline{\text { Resumen de licencia - Texto completo de la licencia }}$ 